\title{
A NECESSARY CONDITION FOR THE EXISTENCE OF SINGULARITY-FREE GLOBAL SOLUTIONS TO NONLINEAR ORDINARY DIFFERENTIAL EQUATIONS*
}

\author{
By GERALD ROSEN (Drexel Institute of Technology)
}

A dilatation invariance argument is employed to derive a necessary condition for the existence of singularity-free global solutions to nonlinear ordinary differential equations associated with a variational principle. It is shown that the necessary condition, in combination with the differential equation itself, is often sufficient to preclude the existence of such a solution.

Consider the generic class of second-order nonlinear ordinary differential equations of the Euler form

$$
y^{\prime \prime} \frac{\partial^{2} F}{\partial y^{\prime 2}}+y^{\prime} \frac{\partial^{2} F}{\partial y \partial y^{\prime}}+\frac{\partial^{2} F}{\partial x \partial y^{\prime}}-\frac{\partial F}{\partial y}=0,
$$

where $F=F\left(x, y, y^{\prime}\right)$ is a prescribed $C^{2}$ real function with respect to its three real arguments and $y=y(x)$ is to be determined as a real singularity-free $C^{2}$ global solution of (1) either for all real $x$ or for all real nonnegative $x$ subject to prescribed boundary conditions at either $x= \pm \infty$ or at $x=0, \infty$. Suppose that $F$ admits a linear decomposition

$$
\begin{gathered}
F=\sum_{(W)} F^{(W)}\left(x, y, y^{\prime}\right), \\
F^{(W)}\left(\lambda x, y, \lambda^{-1} y^{\prime}\right) \equiv \lambda^{W-1} F^{(W)}\left(x, y, y^{\prime}\right)
\end{gathered}
$$

for all real $\lambda>0$, and that the functionals

$$
J^{(W)}[y(x)] \equiv \int_{D} F^{(W)}\left(x, y, y^{\prime}\right) d x
$$

exist for the solution $y(x)$ with $D$ denoting the infinite or semi-infinite $x$ domain; then we have $J^{(W)}\left[y\left(\lambda^{-1} x\right)\right] \equiv \lambda^{W} J^{(W)}[y(x)]$. Hence, as a consequence of the variational principle for (1)

$$
\delta\left(\sum_{|W|} J^{(W)}[y(x)]\right)=0 \Rightarrow\left(\frac{d}{d \lambda} \sum_{\{W\}} J^{(W)}\left[y\left(\lambda^{-1} x\right)\right]\right)_{\lambda-1}=0,
$$

it follows that the relation

$$
\sum_{(W)} W J^{(W)}[y(x)]=0
$$

is a necessary condition for the existence of such a singularity-free global solution $y(x)$. The following examples illustrate that (5) in combination with (1) is often sufficient to preclude the existence of such a solution and thus to obviate well-known but involved computational criteria for existence [1]-[3]. Moreover, the existence of singularity-free global solutions to certain higher-order nonlinear ordinary differential equations (asso-

*Received June 9, 1967. Work supported by a National Science Foundation grant. 
ciated with a variational principle) and to certain nonlinear partial differential equations (associated with a variational principle) can be precluded by similar analysis based on a relation of the form (5) [4].

First EXAMPLE (embracing both Emden and Thomas-Fermi type equations):

$$
x^{\alpha} y^{\prime \prime}+\alpha x^{\alpha-1} y^{\prime}+\gamma x^{\beta} y^{\gamma-1}=0 \quad[0 \leq x<\infty],
$$

where $y(x)$ is nonnegative and $\alpha, \beta, \gamma$ are real constant parameters. We have $F=$ $F^{(\alpha-1)}+F^{(\beta+1)}$ with $F^{(\alpha-1)}=x^{\alpha}\left(y^{\prime}\right)^{2}$ and $F^{(\beta+1)}=-2 x^{\beta} y^{\gamma}$. For a solution such that the functionals (3) are finite, Eq. (5) produces

$$
\int_{0}^{\infty}\left[(\alpha-1) x^{\alpha}\left(y^{\prime}\right)^{2}-2(\beta+1) x^{\beta} y^{\gamma}\right] d x=0 .
$$

Integrating the first term in the integral by parts, using the differential equation, and assuming the boundary condition $\lim _{x \rightarrow 0}\left(x^{\alpha} y y^{\prime}\right)=0$, we find

$$
[(\alpha-1) \gamma-2(\beta+1)] \int_{0}^{\infty} x^{\beta} y^{\gamma} d x=0
$$

which implies that the relation $(\alpha-1) \gamma=2(\beta+1)$ is required for the existence of such a singularity-free global solution. Clearly, for $\gamma$ an even integer this result holds with the nonnegativity of $y(x)$ relaxed. Special cases apply to the Emden equation $(\alpha=2$, $\beta=2, \gamma>0)$, the Thomas-Fermi equation $(\alpha=0, \beta=-1 / 2, \gamma=5 / 2)$, the Bessel equation $(\alpha=1, \beta=-1, \gamma=2)$, the Euler equation $(\alpha=0, \beta=-2, \gamma=2)$, etc.

SeCOND EXAMPLe (an equation of recent interest in elementary particle physics [5], [6]):

$$
x^{2} y^{\prime \prime}+2 x y^{\prime}+x^{2}\left(y^{\gamma-1}-y\right)=0 \quad[0 \leq x<\infty] .
$$

We have $F=F^{(1)}+F^{(3)}$ with $F^{(1)}=x^{2}\left(y^{\prime}\right)^{2}$ and $F^{(3)}=x^{2}\left(y^{2}-[2 / \gamma] y^{\gamma}\right)$. For a solution such that the functionals (3) are finite, Eq. (5) produces

$$
\int_{0}^{\infty} x^{2}\left[\left(y^{\prime}\right)^{2}+3\left(y^{2}-\frac{2}{\gamma} y^{\gamma}\right)\right] d x=0 .
$$

Integrating the first term in the integral by parts and using the differential equation, we find

$$
\int_{0}^{\infty} x^{2}\left[2 y^{2}+\left(1-\frac{6}{\gamma}\right) y^{\gamma}\right] d x=0,
$$

which implies that no solution exists for $\gamma$ an even integer greater or equal to 6 and no nonnegative solution exists for $\gamma \geq 6$.

\section{ReFERENCES}

[1] T. L. Saaty and J. Bram, Nonlinear mathematics, McGraw-Hill, New York, 1964, Chapter 4

[2] M. A. Krasnosel'skii, Topological methods in the theory of nonlinear integral equations, Pergamon Press, Oxford, 1964, Chapters III and VI

[3] E. L. Ortiz, Trans. Amer. Math. Soc. 95, 101 (1960)

[4] G. Rosen, J. of Math. Phys. 7, 2066, 2071 (1966)

[5] Z. Nehari, Proc. Roy. Irish Acad. A62, 117 (1963)

[6] V. P. Sirikov, Soviet Math. Dokl. 6, 1040 (1965) 